
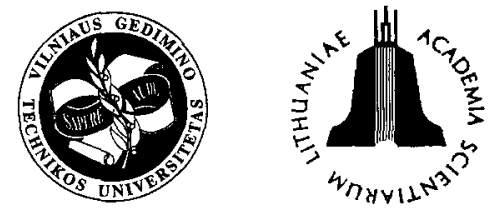

ISSN 1392-3730

JOURNAL OF CIVIL ENGINEERING AND MANAGEMENT

http:/www.vtu.lt/english/editions

2003, Vol LX, No 4, 284-291

\title{
SCHEDULING MODEL OF CONSTRUCTION ACTIVITY WITH TIME COUPLINGS
}

\author{
Zdzisław Hejducki \\ Institute of Building Engineering, Wroctaw University of Technology, \\ Wybrzeże Wyspiańskiego 27,50-370Wrocław, Poland.E-mail: hejducki@pionierib.pwr.wroc.pl
}

Received 22 Aug 2003; accepted 29 Oct 2003

\begin{abstract}
The algorithm of construction work graphic schedule which takes into account the proper sequencing problem has been presented. The algorithm is described step by step, ie the construction work organisation methods with zero couplings between realisation means. The calculation process of "Limit Possible Minimum" (LPM) taking into account technical and organisation limits is also shown. The second part of the paper includes a practical example which presents the method and the optimisation algorithm for the work organisation method with zero couplings among realisation means.
\end{abstract}

Keywords: scheduling model, construction, couplings.

\section{Introduction}

The complexity of realisation processes in construction, where new technological solutions are introduced continuously, requires a continuous improvement of construction work organisation methods. This applies to all stages of the investment process from planning, through design, to realisation. Therefore increasingly sophisticated methods must be developed and applied in practice to solve optimally organisational-technological problems. In construction, the process of planning and scheduling has typically been accomplished using network scheduling techniques. The most common is the critical path method (CPM). Challenges in scheduling method has been offered as a solution to these problems by Arditi 2001a, 200lb, Hegazy 1993, 1999 Johnston 1984. Line of balance (LOB) is a variation of linear scheduling method and is presented by Hamerlink and Rowings (1998), Harris and Ioannou (1998) and provide an algorithm for determining the controling activity path in a linear schedule. Solutions of many particular problems have been presented by Chrzanowski and Johnson (1986), Harris and Ioannou (1998). Solutions of choice of optimal scheduling variants with application of many criteria have been found by Zavadskas $(1997,2002)$. The algorithm of construction work graphic schedule has been presented which takes into account the proper sequencing problem: Afanasjev (1994, 2000), Mrozowicz (1997), Hejducki $(1999,2000,2001)$.

One of the factors determining the effectiveness with which construction works are carried out is their proper sequence taking account the technological and organisational constraints. The problem belongs to the deterministic theory of scheduling tasks and it has permutational character and $n$ ! possible variants. Because of a large number of its possible solutions, the problem is considered to be highly complex computationally. In practice, one can establish a rational sequence in which works are to be carried out by changing the order of works on particular work fronts. However, it would be difficult, or even impossible, to check all the possible realisation variants because of the computational complexity of the combinatorial problems.

Therefore the review division and limitation algorithms enabling the focused surveying of the space of solutions are used. Because of the relatively high computing speed they are more effective, but less accurate than a comprehensive review of realisation variants would be. They allow one to find a suboptimal solution.

To organise building works, methods of division and limitation allowing one to establish a rational sequence of the works are employed. They take into account the specificity of construction processes through an index called "Limit Possible Minimum - (LPM)" which characterises nodes in a tree diagram constructed according to the rules of the division and limitation method.

\section{Elimination and selection rules}

A set of division and limitation algorithms which is used to solve the permutation problem can be characterised by a set of parameters. Among them the selection rule, for determining the search tree's next vertex at which division is to take place, and the elimination rules for the reactivation of the tree's generated nodes - should be singled out. 
The matrix notation of input data is used in construction work organisation methods:

$$
\mathrm{T}=\left[\mathrm{t}_{\mathrm{i}, \mathrm{j}}\right] \quad \mathrm{i}=1, \ldots, \mathrm{m} \mathrm{j}=1, \ldots, \mathrm{m}
$$

where

$t_{i j}$ is a time of carrying out the $\mathrm{j}$-th work on the i-th front.

The notation facilitates the division of works into subsets comprising matrix rows and the formation of tree diagram levels with a dwindling number of nodes. The first level will include $n$ nodes and the last level two nodes.

The elimination and selection rules allow us to move, via calculation procedures, up to the successive levels of the tree diagram and to discard some of the generated vortices. To do it we use an algorithm for determining the nodes' numerical characteristics by means of LPM (Limit Possible Minimum). Since the construction work organisation methods have different properties, the tree's nodes are described in different ways according to the peculiarities of the methods. Algorithms for determining LPM for a construction work organisation method which takes into account couplings between realisation means, couplings between work fronts and their combinations are presented below.

The so-called tree of variants is used to analyse different possible sequences of the work fronts.

In the first stage we must consider $n$ variants. The $\mathrm{j}$-th $(\mathrm{j}=1, \ldots, \mathrm{n})$ variant implies that the $\mathrm{i}$-th variant is realised as the first and the order of other fronts has not been established. For each of the variants we calculate the so-called limit possible time minimum (LPTM) which has the property of being less than time $T_{1}$ calculated for any sequence of the fronts whose order has not been fixed. Thus we know for sure that by expanding further, by establishing the order of the next fronts, the variant with calculated $\mathrm{LPM}_{1}$ we shall not obtain time $T_{1}$ less than $\mathrm{LPM}_{1}$. At the end of stage I we check for which variant the calculated LPM $_{1}$ is the least.

In the second stage we expand the variant for which $\mathrm{LPM}_{1}$ is currently the least. The expansion of the variant with a certain number of rows $s$ whose sequence has been fixed consists in forming new variants $(n-s)$ by one row with a fixed position from the rows whose positions have not been established.

The whole procedure of searching for a sequence of work fronts is completed when the least of the $\mathrm{LPM}_{1} \mathrm{~s}$ calculated so far is realised only by variants whose all fronts have been determined, ie at the very bottom of the variant tree.

Since $\mathrm{LPM}_{1}$ calculated for the above final variants is equal to $T_{1}$ and LPM $_{1}$ cannot be less after the expansion of a given variant, the above procedure will yield all the possible sequences of work fronts with shortest time $\mathrm{T}$ of the realisation of the whole work complex.

\section{Algorithms for establishing sequence in construction work organisation method with zero couplings between realisation means}

We apply a formula for the duration of the realisation of a whole work complex for the continuous use of realisation means

$$
T=\sum_{j=2}^{m} t_{j}^{r}+\sum_{i=1}^{n} t_{i, m},
$$

where $t_{j}^{r}$ is the duration of the expansion of the next $\mathrm{j}$-th partial stream, ie the difference between the time of commencement of the $j$-th partial stream and that of the $\mathrm{j}-1$ th partial stream.

The second summand, ie

$$
\sum_{i=1}^{n} t_{i, m}
$$

does not depend on the sequence in which works are carried out on particular fronts. Thus a search for shortest duration $\mathrm{T}$ of the realisation of a whole complex of works can be limited to the minimum-time optimisation of the first summand, ie

$$
\mathrm{T}_{1}=\sum_{\mathrm{j}=2}^{\mathrm{m}} \mathrm{t}_{\mathrm{j}}^{\mathrm{r}} .
$$

The time of the expansion of $t_{j}^{r}$ depends on the mutual synchronisation of two adjacent partial streams and so it depends on the sequence in which works are carried out on particular work fronts. It can be expressed as follows:

$$
\mathrm{t}_{\mathrm{j}}^{\mathrm{r}}=\max _{1 \leq \mathrm{k} \leq \mathrm{n}}\left[\sum_{\mathrm{i}=1}^{\mathrm{k}} \mathrm{t}_{\mathrm{i}, \mathrm{j}-1}+\sum_{\mathrm{i}=1}^{\mathrm{k}-1} \mathrm{t}_{\mathrm{i}, \mathrm{j}}\right] .
$$

If one solution is sufficient, one needs to test the variant tree only until $\mathrm{LPM}_{1}$ is currently minimum for any fully expanded variant.

To represent fully the way in which the right sequence of carrying out works is established, it is necessary to define the calculation procedure for LPM . For $^{2}$ this purpose one should use modified Johnson's algorithm which allows one to establish the proper sequence of work fronts for two partial streams.

The input data is a two-column matrix:

$$
\begin{aligned}
\mathrm{A}=\left[\mathrm{a}_{\mathrm{i}, \mathrm{k}}\right] \quad \mathrm{i} & =1, \ldots, \mathrm{n} \\
\mathrm{k} & =1,2
\end{aligned}
$$

corresponding to two adjacent partial streams. Then we obtain a three-column matrix:

$$
\begin{aligned}
\mathrm{B}=\left[\mathrm{b}_{\mathrm{i}, \mathrm{k}}\right] \quad \mathrm{i} & =1, \ldots, \mathrm{n} \\
\mathrm{k} & =1,2,3 .
\end{aligned}
$$

TThe first two columns of matrix $B$ consist of the same rows as matrix A does, but in the order established by Johnson's algorithm. The shifted rows' initial numbers bearing information about which work front they come from are in the third column. 
Modified Johnson's algorithm taking account of relationships specific for building works is presented below.

\begin{tabular}{|c|l|}
\hline \multicolumn{1}{|c|}{ Instructions } & \multicolumn{1}{|c|}{ Commentary } \\
\hline $\begin{array}{c}\text { a) Make substitutions: } \\
\text { sg: } 1 ;\end{array}$ & $\begin{array}{l}\text { sg (ds) - number of currently } \\
\text { highest (lowest) free row. }\end{array}$ \\
\hline \multicolumn{1}{|c|}{ max. }
\end{tabular}

Prior to the variant tree testing one should calculate successively auxiliary matrices $B^{j}, j=1, \ldots,(m-1)$. Matrix $\mathrm{B}^{\mathrm{j}}$ is created by applying Johnson's algorithm to data matrix A consisting of columns having numbers $\mathbf{j}$, $(\mathrm{j}+1)$, ie

$$
\begin{aligned}
\mathrm{A}=\left[\mathrm{t}_{\mathrm{i}, \mathrm{j}}\right] \quad \mathrm{i}=1, \ldots, \mathrm{n} \\
\mathrm{k}=1, \mathrm{j}+1 .
\end{aligned}
$$

Now we calculate $\mathrm{LPM}_{1}$ for a case when the sequence of fronts (rows) bearing numbers $\left(\mathrm{u}_{1}, \ldots, \mathrm{u}_{\mathrm{s}}\right), \mathrm{s} \leq \mathrm{n}$ has been established. Let us denote set $\left(u_{1}, \ldots, u_{S}\right)$, consisting of the numbers of the rows whose sequence has been established by $\mathrm{D}$.

\section{Practical example}

In this paper a set of algorithms for scheduling construction work has been presented. As an illustration of

\begin{tabular}{|c|c|}
\hline Instructions & Commentary \\
\hline $\begin{array}{l}\text { a) For each } \mathrm{j}=1, \ldots,(\mathrm{m}-1) \\
\text { create matrix } \\
\mathrm{C}^{\mathrm{j}}=\left[\mathrm{C}_{\mathrm{i}, \mathrm{k}}^{\mathrm{j}}\right] \mathrm{i}=1, \ldots, \mathrm{n} \\
\qquad \mathrm{k}=1,2 \\
\text { as follows: } \\
\text { i) For all indices } \mathrm{i}=1, \ldots \mathrm{s} \text {; } \\
\mathrm{k}=1,2 \text { substitute } \\
\mathrm{C}_{\mathrm{i}, \mathrm{k}}^{\mathrm{j}}=\mathrm{t}_{\mathrm{ui}, \mathrm{j}}+\mathrm{l}-\mathrm{t} \\
\text { ii) Substitute: } \mathrm{p}:=\mathrm{s}+1 ; \\
\text { iii) For all in turn } \mathrm{i}=1, \ldots \mathrm{n} \\
\text { do } \\
\text { if } b_{i, 3}^{j} \varepsilon \mathrm{D}, \text { substitute: } \\
\mathrm{p}:=\mathrm{p}+1 ; \\
C_{p, 1}^{j}=b_{i, k}^{j} \text { for } \mathrm{k}=1,2\end{array}$ & $\begin{array}{l}\text { Matrix } \mathrm{C}^{\mathrm{j}} \text { shows } \\
\text { relationship between } \\
\text { adjacent columns. } \\
\text { First } s \text { rows have } \\
\text { already been } \\
\text { determined. } \\
\text { Introduction of } \\
\text { remaining rows in order } \\
\text { established by auxiliary } \\
\text { matrix } \mathrm{B}^{\mathrm{j}} \text {. }\end{array}$ \\
\hline $\begin{array}{l}\text { b) For all } j=1, \ldots,(m-1) \\
\text { calculate } \\
\mathbf{t}_{j}^{\mathrm{I}}=\max _{1 \leq k \leq n}\left[\sum_{i=1}^{k} c_{i, 1}+\sum_{i=1}^{k-1} c_{1}\right.\end{array}$ & $\begin{array}{l}\text { Period of expansion of } \\
\text { matrix C's second } \\
\text { column relative to the } \\
\text { first one is calculated. }\end{array}$ \\
\hline c) $\mathrm{LPM}_{1}=\sum_{\mathrm{j}=1}^{\mathrm{m}-1} \mathrm{t}_{\mathrm{j}}^{\mathrm{r}}$ & \\
\hline
\end{tabular}
the algorithm for work planning by the work organisation method with zero couplings between realisation means, an investment problem, consisting in the realisation of four structures (A-B-C-D) on which seven technological processes: I - earth work, II - foundation work, III masonry work, IV - concreting work, V - roofing work, VI - plaster work and VII - finishing work are to be carried out, was formulated. Work durations, constituting elements of a work lead time matrix, were determined. A detailed calculation procedure based on the algorithm described above was worked out (Table 1).

\section{Conclusions}

As a result of the calculations, a new realisation sequence A-D-C-B was determined. This realisation sequence ensures a reduction in the task lead time from 260 units to 244 units, ie $6,15 \%$.

The algorithm for scheduling work by the work organisation method with zero couplings between realization means was applied to a practical realisation problem.

A computer system named ORGANISER, which incorporates the presented algorithms and enables their use in construction practice, has been developed. 
Table 1. Sequencing works in work complex by, method with zero couplings between realisation means

Work lead times for particular structures - initial matrix

\begin{tabular}{|c|c|c|c|c|c|c|c|}
\cline { 2 - 8 } \multicolumn{1}{c|}{} & $\begin{array}{c}\text { Earth } \\
\text { work }\end{array}$ & $\begin{array}{c}\text { Foundation } \\
\text { work }\end{array}$ & $\begin{array}{c}\text { Masonry } \\
\text { work }\end{array}$ & $\begin{array}{c}\text { Concreting } \\
\text { work }\end{array}$ & $\begin{array}{c}\text { Roofing } \\
\text { work }\end{array}$ & $\begin{array}{c}\text { Plaster } \\
\text { work }\end{array}$ & $\begin{array}{c}\text { Finishing } \\
\text { work }\end{array}$ \\
\cline { 2 - 8 } & I & II & III & IV & V & VI & VII \\
\hline Object A & 4 & 2 & 26 & 23 & 12 & 8 & 32 \\
\hline Object B & 6 & 2 & 17 & 5 & 5 & 10 & 37 \\
\hline Object C & 3 & 4 & 29 & 22 & 3 & 19 & 39 \\
\hline Object D & 3 & 4 & 20 & 13 & 11 & 13 & 34 \\
\hline
\end{tabular}

Deployment times for particular works

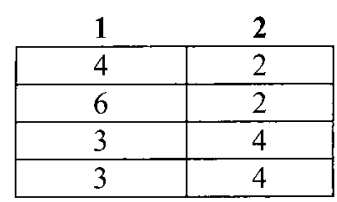

$\mathbf{T r}_{\mathrm{F}}=\mathbf{9}$

\begin{tabular}{|c|c|}
\hline \multicolumn{1}{c}{$\mathbf{4}$} & $\mathbf{5}$ \\
\hline 23 & 12 \\
\hline 5 & 5 \\
\hline 22 & 3 \\
\hline 13 & 11 \\
\hline
\end{tabular}

$\operatorname{Tr}_{\mathrm{R}}=43$

\begin{tabular}{|c|c|}
\multicolumn{1}{c}{} & $\mathbf{3}$ \\
\hline 2 & 26 \\
\hline 2 & 17 \\
\hline 4 & 29 \\
\hline 4 & 20 \\
\hline
\end{tabular}

$\operatorname{tr}_{\mathbf{M}}=\mathbf{2}$

\begin{tabular}{|c|c|}
\multicolumn{1}{c|}{$\mathbf{5}$} & $\mathbf{6}$ \\
\hline 12 & 8 \\
\hline 5 & 10 \\
\hline 3 & 19 \\
\hline 11 & 13 \\
\hline
\end{tabular}

$\operatorname{Tr}_{\mathrm{P}}=12$

\begin{tabular}{|c|c|}
\hline \multicolumn{1}{c}{3} & $\mathbf{4}$ \\
\hline 26 & 23 \\
\hline 17 & 5 \\
\hline 29 & 22 \\
\hline 20 & 13 \\
\hline
\end{tabular}

$\operatorname{Tr}_{\mathrm{C}}=44$

\begin{tabular}{|c|c|}
\hline \multicolumn{1}{c}{6} & 7 \\
\hline 8 & 32 \\
\hline 10 & 37 \\
\hline 19 & 39 \\
\hline 13 & 34 \\
\hline
\end{tabular}

$\operatorname{Tr}_{\mathbf{F}}=8$

Lead time for structure

$$
\begin{gathered}
T=t_{r F}+t_{r M}+t_{r C}+t_{r R}+t_{r P}+t_{r F}+\Sigma t_{i m 9 w 0} \\
T S=9+2+44+43+12+8+142=260 \text { days }
\end{gathered}
$$

Matrices reconstructed according to Johnson's algorithm

\begin{tabular}{|c|c|}
\hline \multicolumn{1}{|l}{ ' } & 2' \\
\hline 3 & 4 \\
\hline 3 & 4 \\
\hline 4 & 2 \\
\hline 6 & 2 \\
\hline
\end{tabular}

\begin{tabular}{|l|l|}
\hline \multicolumn{1}{|c}{ 2' } & 3' \\
\hline 2 & 17 \\
\hline 2 & 26 \\
\hline 4 & 20 \\
\hline 4 & 29 \\
\hline
\end{tabular}

\begin{tabular}{|c|c|}
\multicolumn{1}{|c}{$\mathbf{3}^{\prime}$} & $\mathbf{4}^{\prime}$ \\
\hline 29 & 22 \\
\hline 26 & 23 \\
\hline 20 & 13 \\
\hline 17 & 5 \\
\hline
\end{tabular}

\begin{tabular}{|c|c|}
\multicolumn{1}{|c}{$\mathbf{4}^{\prime}$} & $\mathbf{5}$ \\
\hline 23 & 12 \\
\hline 13 & 11 \\
\hline 5 & 5 \\
\hline 22 & 3 \\
\hline
\end{tabular}

\begin{tabular}{|c|c|}
\multicolumn{1}{c}{5} & $\mathbf{6}$ \\
\hline 3 & 19 \\
\hline 5 & 10 \\
\hline 11 & 13 \\
\hline
\end{tabular}

\begin{tabular}{|c|c|}
\hline \multicolumn{1}{|c}{$\boldsymbol{6}^{\mathbf{7}}$} & $\mathbf{7}$ \\
\hline 8 & 32 \\
\hline 10 & 37 \\
\hline 13 & 34 \\
\hline
\end{tabular}


Matrices with succesively determined rows:

\begin{tabular}{|c|c|c|c|c|c|c|c|c|c|c|c|c|}
\hline \multirow[b]{2}{*}{$\mathbf{A}$} & \multicolumn{2}{|l|}{1} & 2 & \multicolumn{2}{|c|}{3} & \multicolumn{2}{|c|}{4} & \multicolumn{2}{|c|}{5} & \multicolumn{2}{|c|}{6} & 7 \\
\hline & 4 & \multicolumn{2}{|c|}{2} & \multicolumn{2}{|c|}{26} & \multicolumn{2}{|c|}{23} & \multicolumn{2}{|c|}{12} & \multicolumn{2}{|c|}{8} & 32 \\
\hline & 3 & 4 & 2 & 17 & 29 & 22 & 13 & 11 & 3 & 19 & 10 & 37 \\
\hline & 3 & 4 & 4 & 20 & 20 & 13 & 5 & 5 & 5 & 10 & 13 & 34 \\
\hline & 6 & 2 & 4 & 29 & 17 & 5 & 22 & 3 & 11 & 13 & 19 & 39 \\
\hline
\end{tabular}

$\mathrm{GMM}_{\mathrm{A}}^{\mathrm{I}}=6+2+34+35+12+8+142=\mathbf{2 3 9}$

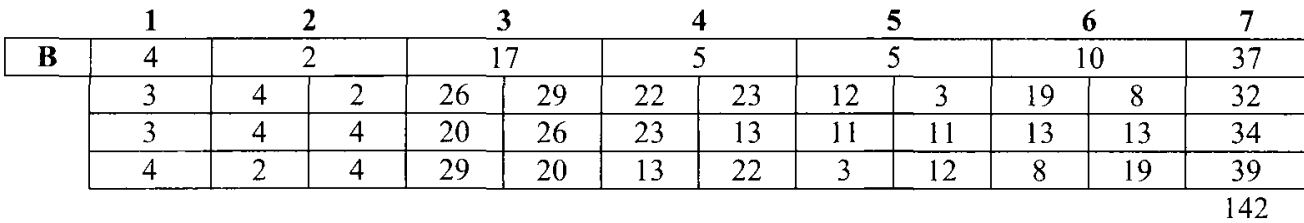

$\mathrm{GMM}_{\mathrm{B}}^{\mathrm{I}}=7+2+45+35+5+10+142=\mathbf{2 4 6}$

\begin{tabular}{|c|c|c|c|c|c|c|c|c|c|c|c|c|}
\hline \multirow{3}{*}{$\mathrm{C}$} & 1 & \multicolumn{2}{|c|}{2} & \multicolumn{2}{|c|}{3} & \multicolumn{2}{|c|}{4} & \multicolumn{2}{|c|}{5} & \multicolumn{2}{|c|}{6} & 7 \\
\hline & 3 & \multicolumn{2}{|c|}{4} & \multicolumn{2}{|c|}{29} & \multicolumn{2}{|c|}{22} & \multicolumn{2}{|c|}{3} & \multicolumn{2}{|c|}{19} & 39 \\
\hline & 3 & 4 & 2 & 17 & 26 & 23 & 23 & 12 & 5 & 10 & 8 & 32 \\
\hline & 4 & 2 & 2 & 26 & 20 & 13 & 13 & 11 & 11 & 13 & 10 & 37 \\
\hline & 6 & 2 & 4 & 20 & 17 & 5 & 5 & 5 & 12 & 8 & 13 & 34 \\
\hline
\end{tabular}

$\mathrm{GMM}_{\mathrm{C}}^{\mathrm{I}}=6+4+34+43+3+19+142=\mathbf{2 5 1}$

\begin{tabular}{|c|c|c|c|c|c|c|c|c|c|c|c|c|}
\hline \multirow{3}{*}{ D } & \multicolumn{2}{|l|}{1} & 2 & \multicolumn{2}{|c|}{3} & \multicolumn{2}{|c|}{4} & \multicolumn{2}{|c|}{5} & \multicolumn{2}{|c|}{6} & 7 \\
\hline & 3 & \multicolumn{2}{|c|}{4} & \multicolumn{2}{|c|}{20} & \multicolumn{2}{|c|}{13} & \multicolumn{2}{|c|}{11} & \multicolumn{2}{|c|}{13} & 34 \\
\hline & 3 & 4 & 2 & 17 & 29 & 22 & 23 & 12 & 3 & 19 & 8 & 32 \\
\hline & 4 & 2 & 2 & 26 & 26 & 23 & 5 & 5 & 5 & 10 & 10 & 37 \\
\hline & 6 & 2 & 4 & 29 & 17 & 5 & 22 & 3 & 12 & 8 & 19 & 39 \\
\hline
\end{tabular}

$\mathrm{GMM}_{\mathrm{D}}^{\mathrm{I}}=6+4+40+35+11+13+142=\mathbf{2 5 1}$

Matrices constructed at intermediate stages:

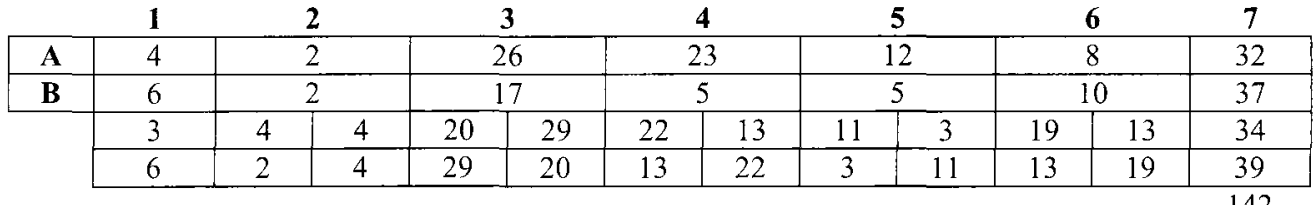

$\mathrm{GMM}_{\mathrm{AB}}^{\mathrm{II}}=9+2+44+35+12+8+142=\mathbf{2 5 2}$

\begin{tabular}{|c|c|c|c|c|c|c|c|c|c|c|c|c|}
\hline & 1 & \multicolumn{2}{|c|}{2} & \multicolumn{2}{|c|}{3} & \multicolumn{2}{|c|}{4} & \multicolumn{2}{|c|}{5} & \multicolumn{2}{|c|}{6} & 7 \\
\hline $\mathbf{A}$ & 4 & \multicolumn{2}{|c|}{2} & \multicolumn{2}{|c|}{26} & \multicolumn{2}{|c|}{23} & \multicolumn{2}{|c|}{12} & \multicolumn{2}{|c|}{8} & 32 \\
\hline $\mathrm{C}$ & 3 & \multicolumn{2}{|c|}{4} & \multicolumn{2}{|c|}{29} & \multicolumn{2}{|c|}{22} & \multicolumn{2}{|c|}{3} & \multicolumn{2}{|c|}{19} & 39 \\
\hline & 3 & 4 & 2 & 17 & 20 & 13 & 13 & 11 & 11 & 13 & 10 & 37 \\
\hline & 6 & 2 & 4 & 20 & 17 & 5 & 5 & 5 & 10 & 12 & 13 & 34 \\
\hline
\end{tabular}

$\mathrm{GMM}_{\mathrm{AC}}^{\mathrm{Il}}=6+2+34+43+12+8+142=\mathbf{2 4 7}$ 


\begin{tabular}{|c|c|c|c|c|c|c|c|c|c|c|c|c|}
\hline & 1 & \multicolumn{2}{|c|}{2} & \multicolumn{2}{|c|}{3} & \multicolumn{2}{|c|}{4} & \multicolumn{2}{|c|}{5} & \multicolumn{2}{|c|}{6} & 7 \\
\hline$\overline{\mathbf{A}}$ & 4 & \multicolumn{2}{|c|}{2} & \multicolumn{2}{|c|}{26} & \multicolumn{2}{|c|}{23} & \multicolumn{2}{|c|}{12} & \multicolumn{2}{|c|}{8} & 32 \\
\hline D & 3 & \multicolumn{2}{|c|}{4} & \multicolumn{2}{|c|}{20} & \multicolumn{2}{|c|}{13} & \multicolumn{2}{|c|}{11} & \multicolumn{2}{|c|}{13} & 34 \\
\hline & 3 & 4 & 2 & 17 & 29 & 22 & 5 & 5 & 3 & 19 & 10 & 37 \\
\hline & 6 & 2 & 4 & 29 & 17 & 5 & 22 & 3 & 5 & 10 & 19 & 39 \\
\hline
\end{tabular}

$$
\mathrm{GMM}_{\mathrm{AD}}^{\mathrm{II}}=6+2+34+35+15+8+142=\mathbf{2 4 6}
$$

Determining sequence of rows:

\begin{tabular}{|c|c|c|c|c|c|c|c}
\multicolumn{1}{c}{$\mathbf{1}$} & $\mathbf{2}$ & $\mathbf{3}$ & $\mathbf{4}$ & $\mathbf{5}$ & $\mathbf{6}$ & $\mathbf{7}$ \\
\hline A & 4 & 2 & 26 & 23 & 12 & 8 & 32 \\
\hline C & 3 & 4 & 29 & 22 & 3 & 19 & 39 \\
\hline B & 6 & 2 & 17 & 5 & 5 & 10 & 37 \\
\hline D & 3 & 4 & 20 & 13 & 11 & 13 & 34 \\
\hline
\end{tabular}

$$
\mathrm{GMM}_{\mathrm{ACBD}}^{\mathrm{III}}=8+2+42+43+12+8+142=\mathbf{2 5 7}
$$

\begin{tabular}{|c|c|c|c|c|c|c|c}
\multicolumn{2}{c}{$\mathbf{1}$} & $\mathbf{2}$ & $\mathbf{3}$ & $\mathbf{4}$ & $\mathbf{5}$ & $\mathbf{6}$ & $\mathbf{7}$ \\
\hline $\mathbf{A}$ & 4 & 2 & 26 & 23 & 12 & 8 & 32 \\
\hline C & 3 & 4 & 29 & 22 & 3 & 19 & 39 \\
\hline D & 3 & 4 & 20 & 13 & 11 & 13 & 34 \\
\hline B & 6 & 2 & 17 & 5 & 5 & 10 & 37 \\
\hline
\end{tabular}

\begin{tabular}{|c|c|c|c|c|c|c|c|}
\hline & 1 & 2 & 3 & 4 & 5 & 6 & 7 \\
\hline $\mathbf{A}$ & 4 & 2 & 26 & 23 & 12 & 8 & 32 \\
\hline D & 3 & 4 & 20 & 13 & 11 & 13 & 34 \\
\hline B & 6 & 2 & 17 & 5 & 5 & 10 & 37 \\
\hline $\mathrm{C}$ & 3 & 4 & 29 & 22 & 3 & 19 & 39 \\
\hline
\end{tabular}

$$
\mathrm{GMM}_{\mathrm{ACDB}}^{\mathrm{III}}=6+2+34+43+12+8+142=\mathbf{2 4 4}
$$

\begin{tabular}{|c|c|c|c|c|c|c|c|}
\hline & 1 & 2 & 3 & 4 & 5 & 6 & 7 \\
\hline $\mathbf{A}$ & 4 & 2 & 26 & 23 & 12 & 8 & 32 \\
\hline D & 3 & 4 & 20 & 13 & 11 & 13 & 34 \\
\hline $\mathrm{C}$ & 3 & 4 & 29 & 22 & 3 & 19 & 39 \\
\hline B & 6 & 2 & 17 & 5 & 5 & 10 & 37 \\
\hline
\end{tabular}

$\mathrm{GMM}_{\mathrm{ADBC}}^{\mathrm{III}}=8+2+51+35+15+8+142=\mathbf{2 6 1}$

$\mathrm{GMM}^{\mathrm{III}}{ }_{\mathrm{ADCB}}=6+2+39+37+15+8+142=\mathbf{2 4 9}$ 


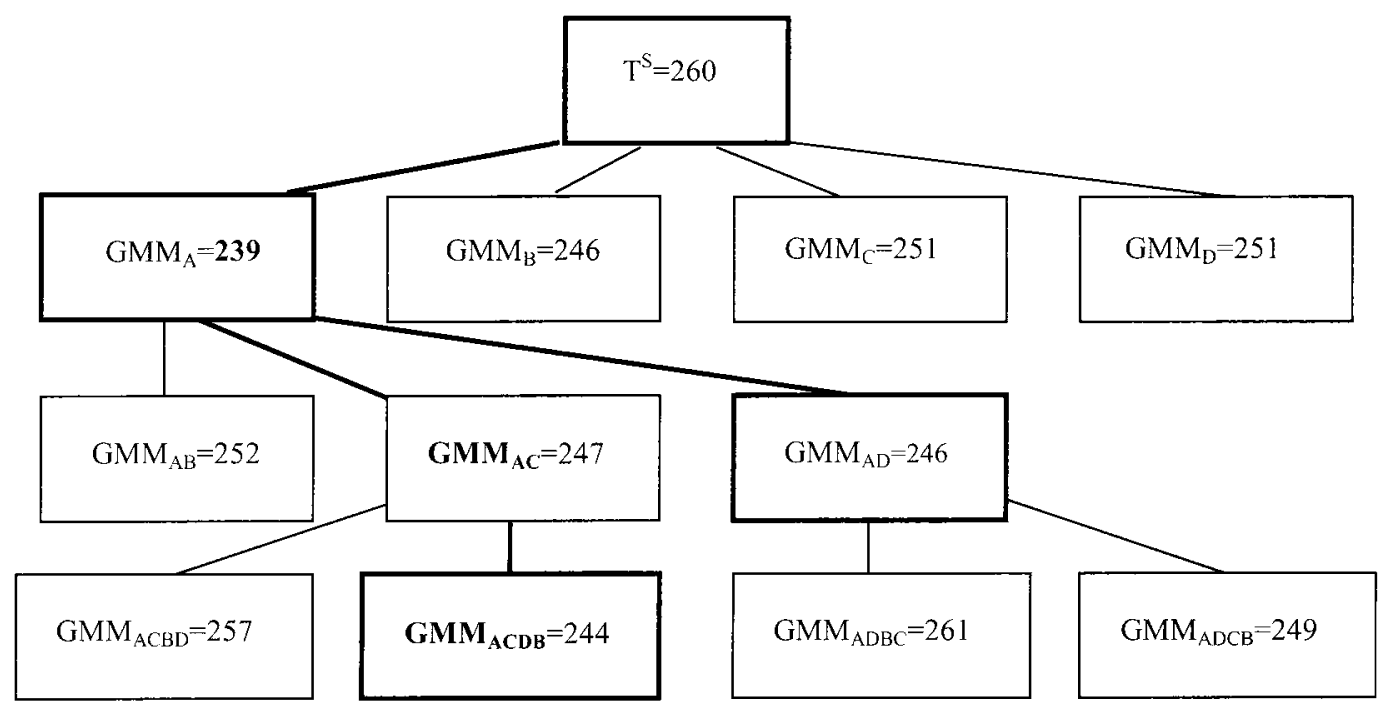

Fig 1. Variant tree

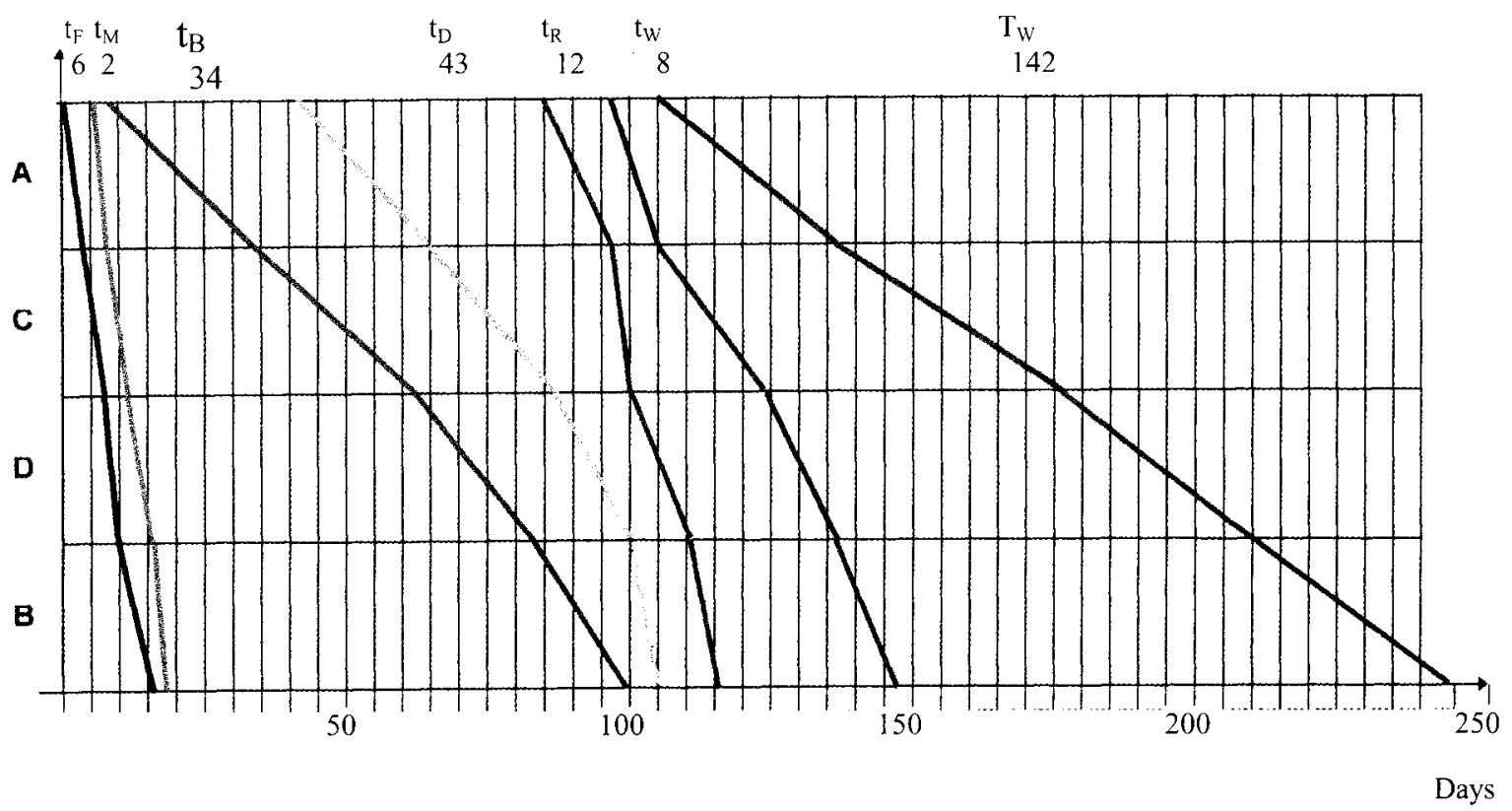

Legend:

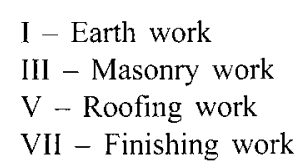

II - Foundation work

IV - Concreting work

VI - Plaster work

Fig 2. Cyclogram of realisation of structures for method with zero couplings between realization means 


\section{References}

1. Afanasev, V. A. Taking into account duration for transformation scheduling with stream complex application (Учет затрат времени на преобразование строительных организаций при формировании комплексов потоков). Present Problems of Civil Engineering, St. Petersburg, 1994 (in Russian).

2. Afanasev, V. A.; Afanasev, A. V. Stream organization of works in civil engineering (Поточная организация работ в строительстве). St. Petersburg, 2000 (in Russian).

3. Arditi, D.; Tokdemir, O. B. \& Suh, K. Scheduling system for repetitive unit construction using line-of-balance technology. Department of Civil and Architectural Engineering, Illinois Institute of Technology. Dept of Civil Engineering, Honan University, Honam, South Korea, 2001a.

4. Arditi, D.; Tokdemir, O. B. \& Suh, K. Effect of learning on line-of-balance scheduling. International Journal of Project Management, $200 \mathrm{lb}$ (in press).

5. Chrzanowski, Jr. E. N., and Johnston, d. W. Application of linear construction. Journal of Construction Engineering, 112(4), 1986, p. 476-491.

6. Hamerlink, D. J.; Rowings, J. E. Linear scheduling model: Development of controlling activity path. Journal of Construction Engineering and Management, 124(4), 1998, p. 266-268.

7. Harris, R. B.; Ioannon, P. G. Scheduling projects with repeating activities. Journal of Construction Engineering and Management, 124(4), 1998, p. 269-278.
8. Hegazy, T. Optimization of construction time - cost tradeoff analysis using genetic algorithms. Canadian Journal of Civil Engineering, 26, 1999, p. 685-697.

9. Hegazy, T.; Moselhi, O. \& Fazio, P. BAL: an algorithm for scheduling and control of linear projects. 1993.

10. AACE Transactions, C. 8, 8.1-8.14.

11. Hejducki, Z.; Mrozowicz, J. Synchronization problems of construction work complexes (Проблема синхронизации комплексов строительных работ), International Symposium, Moscow, Russia, 1999 (in Russian).

12. Hejducki, Z. Construction work organisation methods taking into account zero values of diagonal couplings. IV Internationalni Sympozijum iz Project Managementa, Beograd, 2000.

13. Hejducki, Z.; Mrozowicz, J. Stream methods of construction work organisation, Engineering, Construction and Architectural Management 8/1/200, 2001

14. Johnston, D. W. Linear scheduling method for highway construction. J. Constr. Div., Am. Soc. Civ. Eng., 107(2), 1984, p. 247-261.

15. Mrozowicz, J. Methods of organizing construction processes, which take into account time couplings. Dolnoślaskie Wydawnictwo Edukacyjne, Wrocław, 1997 (in Polish).

16. Zavadskas, E. K. Multiple criteria solutions in construction (Mehrkriterielle Entscheidungen im Bauwesen). Vilnius: Technika, 2000 (in German).

17. Zavadskas, E. K.; Kaklauskas, A.; Turskis, Z. Multicriteria decision-making system for building refurbishment. Civil Engineering (Statyba). Vilnius: Technika, No 4(12), 1997, p. 62-68 (in Lithuanian) 